\title{
MULTIFUNCTIONAL VEHICLE STRUCTURAL HEALTH MONITORING OPPORTUNITIES WITH PIEZOELECTRIC WAFER ACTIVE SENSORS
}

\author{
Victor Giurgiutiu, PhD, Senior Member AIAA \\ University of South Carolina, Columbia, SC 29208, victorg@sc.edu \\ John H. Barnes and Lt. Dustin Thomas \\ Air Force Research Laboratory, WPAFB, OH 45433
}

\begin{abstract}
The capability of embedded piezoelectric wafer active sensors (PWAS) to perform in-situ nondestructive evaluation (NDE) is explored. Laboratory tests are used to prove that PWAS can satisfactorily perform Lamb wave transmission and reception. Subsequently, crack detection in an aircraft panel with the pulseecho method is illustrated. For large area scanning, a $P W A S$ phased array is used to create the embedded ultrasonics structural radar (EUSR). Opportunities for implementation into Air Force vehicles and the research needs are also discussed.
\end{abstract}

\section{INTRODUCTION}

Structural Health Monitoring is a major component of the Integrated Vehicle Health Monitoring thrust within the Robust Aerospace Vehicle concept, as jointly described by AFRL, ONR, NASA, and industry ${ }^{1}$. This very large research area brings together a variety of disciplines. Current research interests in this area reside with: (a) development of embedded active sensors; (b) processing of active sensor data and diagnostics of the health of the structure; (c) prognostics of the remaining structural life. For the health monitoring of an actual structure, networks of embedded active sensors are envisaged. Such sensory networks would be clustered around structural "hot spots". The networks could be wirelessly connected to a data repository and knowledge base site (Figure 1).

Embedded nondestructive evaluation (NDE) is an emerging technology that will allow transitioning the methods of conventional ultrasonics to embedded systems structural health monitoring (SHM) such as those envisioned for the Integrated Vehicle Health Management (IVHM). SHM for IVHM- requires the development of small, lightweight, inexpensive, unobtrusive, minimally invasive sensors to be embedded in the airframe with minimum weight penalty and at affordable costs ${ }^{1}$. Such sensors should be able to scan the structure and identify the presence of defects and incipient damage.

Current ultrasonic inspection of thin wall structures (e.g., aircraft shells, storage tanks, large pipes, etc.) is a time consuming operation that requires meticulous through-the-thickness $\mathrm{C}$-scans over large areas. One method to increase the efficiency of thin-wall structures inspection is to utilize guided waves (e.g., Lamb waves) instead of the conventional pressure waves ${ }^{2}$. Guided waves propagate along the mid-surface of thin-wall plates and shallow shells. They can travel at relatively large distances with very little amplitude loss and offer the advantage of large-area coverage with a minimum of installed sensors ${ }^{3,4}$. Guided Lamb waves have opened new opportunities for cost-effective detection of damage in aircraft structures ${ }^{5}$, and a large number of papers have recently been published on this subject ${ }^{6}$. Traditionally, guided waves have been generated by impinging the plate obliquely with a tone-burst from a relatively large ultrasonic transducer. Snell's law ensures mode conversion at the interface, hence a combination of pressure and shear waves are simultaneously generated into the thin plate. However, conventional Lamb-wave probes (wedge and comb transducers) are relatively too heavy and expensive to be considered for widespread deployment on an aircraft structure as part of a SHM system. Hence, a different type of sensors than the conventional ultrasonic transducers is required for the SHM systems.

Keilers and Chang $^{7}$ were among the first to experiment with built-in piezoelectrics for damage detection based on wave-propagation ${ }^{8}$ Other investigators ${ }^{9}$ have recently explored the generation of Lamb-waves on the same principles. Giurgiutiu and Zagrai $^{10}$ identified these new devices as piezoelectric wafer active sensors (PWAS) and developed a method for their systematic characterization. PWAS are inexpensive, non-intrusive, un-obtrusive, and minimally invasive devices that can be surface-mounted on existing structures inserted between the layers of lap joints, or inside composite materials. 


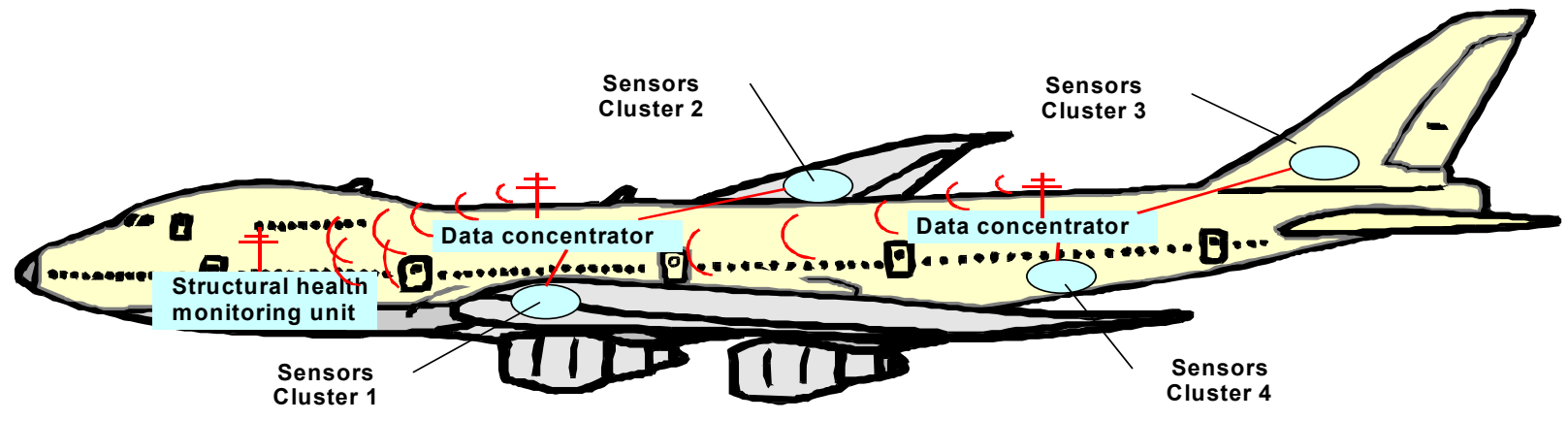

Figure 1 General concept of a sensor-array aircraft structural health monitoring system

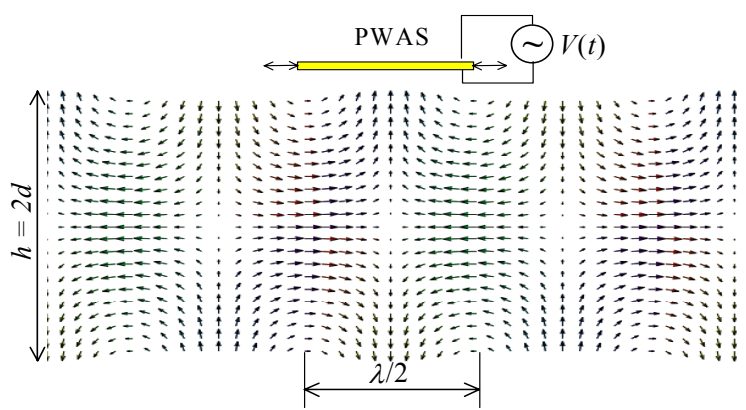

(b)

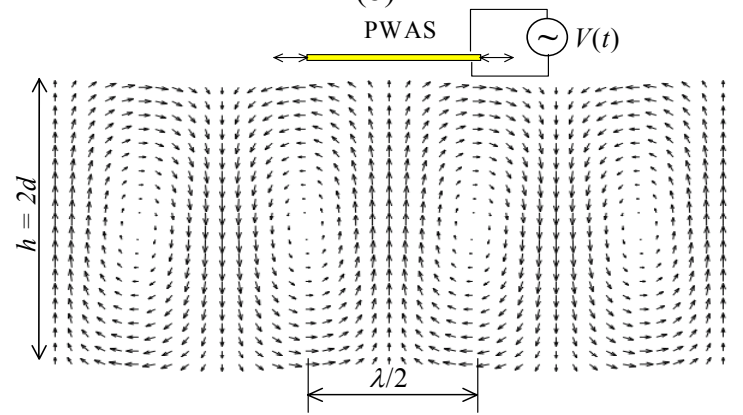

(c)

Figure 2 PWAS interaction with $S_{0}$ and $A_{0}$ Lamb modes

PWAS weight around $68 \mathrm{mg}$, are $0.2 \mathrm{~mm}$ thick, and cost $\$ 7$ each. PWAS operated on the piezoelectric principle that couples the electrical and mechanical variables in the material (mechanical strain, $S_{i j}$, mechanical stress, $T_{k l}$, electrical field, $E_{k}$, and electrical displacement $D_{j}$ ) in the form:

$$
\begin{aligned}
& S_{i j}=s_{i j k l}^{E} T_{k l}+d_{k i j} E_{k} \\
& D_{j}=d_{j k l} T_{k l}+\varepsilon_{j k}^{T} E_{k},
\end{aligned}
$$

where $s_{i j k l}^{E}$ is the mechanical compliance of the material measured at zero electric field $(E=0), \varepsilon_{j k}^{T}$ is the dielectric permittivity measured at zero mechanical stress $(T=0)$, and $d_{k i j}$ represents the piezoelectric coupling effect. For embedded NDE applications, PWAS couple their in-plane motion, excited by the applied oscillatory voltage through the piezoelectric effect, with the Lamb-waves particle motion on the material surface. Lamb waves can be either quasi-axial $(\mathrm{S} 0, \mathrm{~S} 1, \mathrm{~S} 2, \ldots)$, or quasi-flexural $(\mathrm{A} 0, \mathrm{~S} 1, \mathrm{~S} 2, \ldots)$ as shown in Figure 2. PWAS probes can act as both exciters and sensors of the elastic Lamb waves traveling in the material.

For embedded NDE, PWAS can be used as both active and passive probes. Thus, they address four IVHM-SHM needs ${ }^{11}$ :

1) Far-field damage detection using pulse-echo and pitch-catch methods

2) Near-field damage detection using highfrequency impedance method

3) Acoustic emission monitoring of crack initiation and growth

4) Low-velocity impact detection

Diamanti et al. ${ }^{12}$ presented work on a system of piezoceramic PbZrTi (PZT) actuators that could be used for in-situ delamination detection of a composite structure. They used rectangular PZT strips in a phased array to generate low-frequency guided anti-symmetric Lamb waves (Figure 3). Damping material was applied between the PZT strips to significantly reduce the "noise" between the actuators. Using at least four of these PZT actuators in an array, a damage area of $20 \mathrm{x}$ $20 \mathrm{~mm}^{2}$ can be observed. However, the size and the extent of damage to the structure were not accurately predicted.

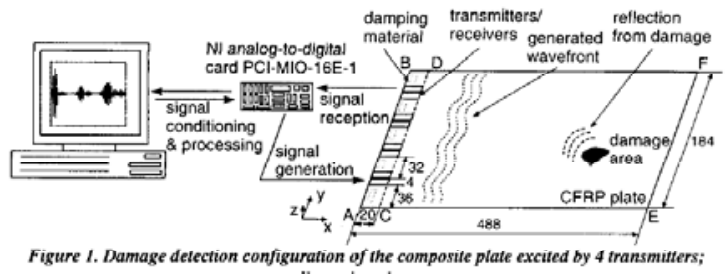

dimensions in $\mathrm{mm}$.

Figure 3 Diamanti et al [17] experiments.

Ghoshal et al. ${ }^{13}$ have developed a model of asymmetric wave propagation in a panel to better understand their physical characteristics. They started by modeling acoustic emission and active wave propagation 
analytically. Then, they analytically modeled a PZT so that damage in the previous models could be detected from dynamic strain measurements. Finally, they used a model of a fiberglass plate to build a simulation of the system.Since the models are analytical in nature, they have not yet been able to model cracks and other flaws in the fiberglass plate.

Guy et al. ${ }^{14}$ was able to relate the influence of a material's mechanical property upon the characteristics of guided lamb waves. They considered two different composite materials for their study, Carbon Fibers Reinforced Plate (CFRP) and Glass Reinforced Plate (GRP). For each composite material, they produced a reproducible low velocity impact to create a defect. For the case of the CFRP, disbonds were created at the impact point. However, large cracked areas were observed around the GRP's impact point. These defects were verified using classical NDI techniques. After characterizing the defects, they induced lamb waves on the plates using PZT discs in a pitch-catch configuration. Interestingly, they found that symmetric lamb wave modes are more sensitive to the delaminations of the CFRP, but the asymmetric lamb wave modes are more sensitive to the cracking induced in the GRP.

Peairs, Park, and Inman ${ }^{15}$ presented research on using PZT wafers for impedance method health monitoring. This method ${ }^{16}$ measures the mechanical impedance of the structure through the real part of the electrical impedance of a PZT wafer attached to the structure. They built a low-cost simple circuit to measure the impedance across the PZT wafer, as the input voltage divided by the current. The output from this circuit is transformed from the time domain to the frequency domain using an FFT analyzer. Experiments compared this with the conventional impedance analyzer. This technology is a step toward the practical use of PZT wafers as a health monitoring method.

Walbrink et al. ${ }^{17}$ attempted to employ PZT wafers to detect cracks at the joints of boons in dragline structures (space truss of cylindrical bars). In order to ascertain the feasibility of applying ultrasonic waves to find cracks in a complex structure, they established a finite element model of ultrasonic waves on this intricate geometry. They found that if the length of the crack is approximately the same as the wavelength, then the diffraction from the crack is more easily distinguishable. Though this work indicates that PZT wafers could induce ultrasonic waves, experimental validation still needs to be done.

It is apparent that the PWAS operation is different from the operation of conventional ultrasonic probes. Not only are PWAS much smaller and cheaper than conventional ultrasonic probes, but also their principles of operation are fundamentally different:

(a) PWAS achieve Lamb wave excitation and sensing through surface "pinching" (in-plane strains), while conventional ultrasonic probes excite through surface "tapping" (normal stress).

(b) PWAS are strongly coupled with the structure and follow the structural dynamics, while conventional ultrasonic probes are relatively free from the structure and follow their own dynamics.

(c) PWAS are non-resonant wide-band devices, while conventional ultrasonic probes are narrow-band resonators.

The main advantage of PWAS over conventional ultrasonic probes lies in their small size, lightweight, low profile, and small cost. In spite of their small size, these novel devices are able to replicate many of the functions of the conventional ultrasonic probes, as proven by the proof-of-concept laboratory demonstrations.

\section{PWAS GENERATED LAMB WAVES}

The basic principles of Lamb wave generation and detection by PWAS probes were first verified on simple laboratory experiments. A 1.6-mm thick, 2024aluminum alloy rectangular plate $(914 \mathrm{~mm} \times 504 \mathrm{~mm} \times$ $1.6 \mathrm{~mm}$ ) was instrumented with eleven 7-mm sq., 0.2mm thick PWAS (American Piezo Ceramics Inc., APC850) placed on a rectangular grid. With this setup, we verified that:

(a) Lamb waves can be satisfactorily generated and detected with PWAS

(b) omnidirectional transmission is achieved

(c) signals are strong enough and attenuation is sufficiently low for echoes to be detected

The proof of these attributes is especially important for PWAS, are at least an order of magnitude smaller and lighter than conventional ultrasonic transducers, and hence utilize much lower power.

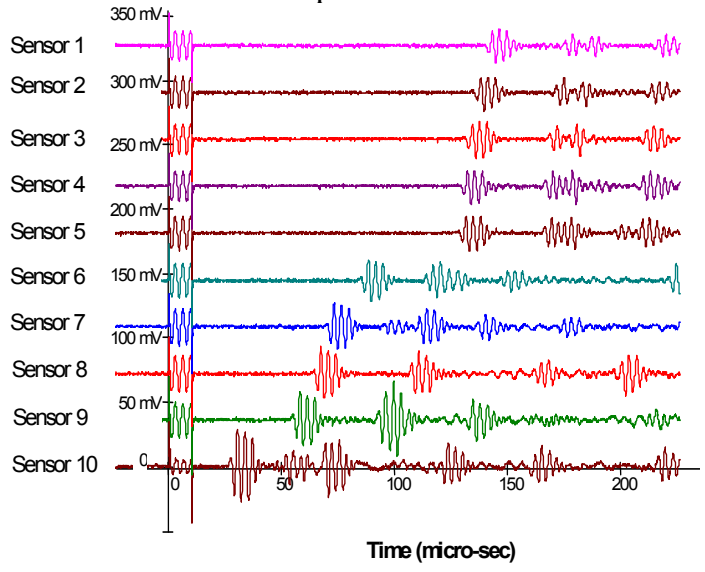

Figure 4 Reception signals on PWAS \#1 through 10

To prove that the Lamb waves excited by PWAS are omnidirectional, we used one PWAS (\#11) as 
transmitter and the other PWAS (\#1-10) as receivers. The signals observed in this investigation are shown in Figure 4a. In each row, the electromagnetic coupling of the 'initial bang' is shown around the origin. Then, the first wave package corresponding to the wave received from the transmitter PWAS is seen, followed by other wave packages corresponding to reflections from the plate edges. The time difference between the initial bang and the wave-package arrival represents the time-of-flight (TOF). The TOF is consistent with the distance traveled by the wave. A straight-line correlation $\left(R^{2}=99.99 \%\right)$ between TOF and distance gave the experimental group velocity, $c_{g}=5.446 \mathrm{~km} / \mathrm{s}$. The theoretical value should be $5.440 \mathrm{~km} / \mathrm{s}$. Very good accuracy is observed $(0.1 \%$ speed detection error). Thus, we proved that PWAS-generated Lamb waves propagate are "loud and clear", propagate omnidirectionally, and correlate well with the theory.

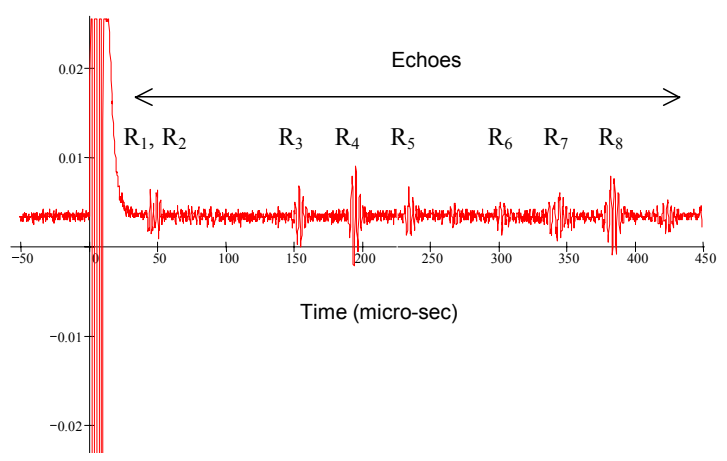

(a)

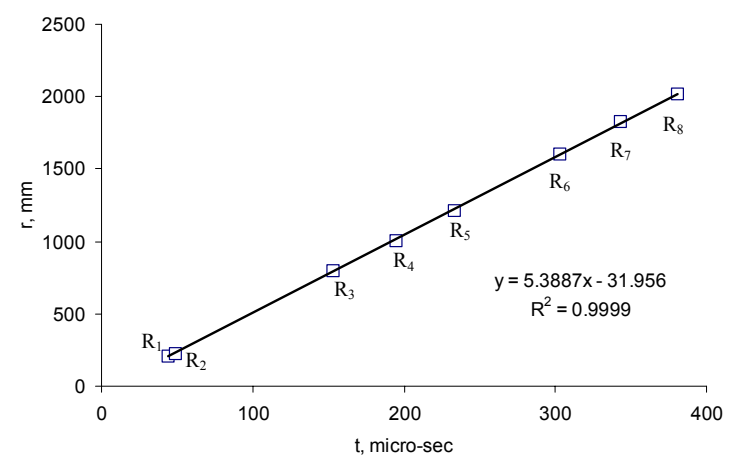

(b)

Figure 5 Pulse-echo method applied to PWAS \#11: (a) the excitation and echo signals; (b) correlation of path length with time of flight.

\section{PULSE-ECHO WITH PWAS}

PWAS \#11 was used for the demonstration of pulseecho capabilities. Figure 5 shows that the sensor \#11 signal has two distinct zones: (i) the initial bang, during which the PWAS \#11 acts as transmitter; and (ii) the echoes zone, containing wave packs reflected by the plate boundaries and sent back to PWAS \#11.
These echoes were processed to evaluate the pulse-echo capabilities of the method. Since the wave generated by the initial bang underwent multiple reflections from the plate edges, each of these reflections had a different path length.

A plot of the TOF of each echo vs. its path length is given in Figure 5b. The straight line fit has a very good correlation $\left(\mathrm{R}^{2}=99.99 \%\right)$ and the corresponding group velocity is $5.389 \mathrm{~km} / \mathrm{s}$, i.e., within $1 \%$ of the theoretical value of $5.440 \mathrm{~km} / \mathrm{s}$. Note that loud and clear echoes were recorded from over 2,000 $\mathrm{mm}$ distance, which is remarkable for such small ultrasonic devices. PWAS are capable of transmitting and receiving pulse-echo signals of remarkable strength and clarity.

\section{PWAS CRACK DETECTION}

Wave propagation experiments were conducted on an aircraft panel to illustrate crack detection through the pulse-echo method. The panel has a typical aircraft construction, featuring a vertical splice joint and horizontal stiffeners. Figure 6 shows three photographs of PWAS installation on increasingly more complex structural regions of the panel. Adjacent to the photographs are the PWAS signals. All the experiments used only one PWAS, operated in pulse-echo mode. The PWAS was placed in the same relative location, i.e., at $200 \mathrm{~mm}$ to the right of the vertical row of rivets. The first row of Figure 6 shows the situation with the lowest complexity, in which only the vertical row of rivets is present in the far left. The signal to the right of this photograph shows the initial bang (centered at around 5.3 micro-sec) and multiple reflections from the panel edges and the splice joint. The echoes start to arrive at approximately $60 \mu \mathrm{s}$. The second row of Figure 6 shows the vertical row of rivets in the far left and, in addition, a horizontal double row of rivets stretching towards the PWAS. The signal to the right shows that, in addition to the multiple echoes from the panel edges and the splice, the PWAS also receives backscatter echoes from the rivets located at the beginning of the horizontal row.

These backscatter echoes are visible at around $42 \mu$ s. The third row in Figure 6 shows a region of the panel similar to that presented in the previous row, but having an additional feature: a simulated crack (12.7-mm EDM hairline slit) emanating from the first rivet hole in the top horizontal row. The signal at the right of this photo shows features similar to those of the previous signal, but somehow stronger at the $42 \mu$ s position. The features at $42 \mu$ s correspond to the superposed reflections from the rivets and from the crack. The detection of the crack seems particularly difficult because the echoes from the crack and from the rivets are superposed. This difficulty was resolved by using the differential signal method, i.e., subtracting the signal presented in the second row from the signal presented in the third row. 

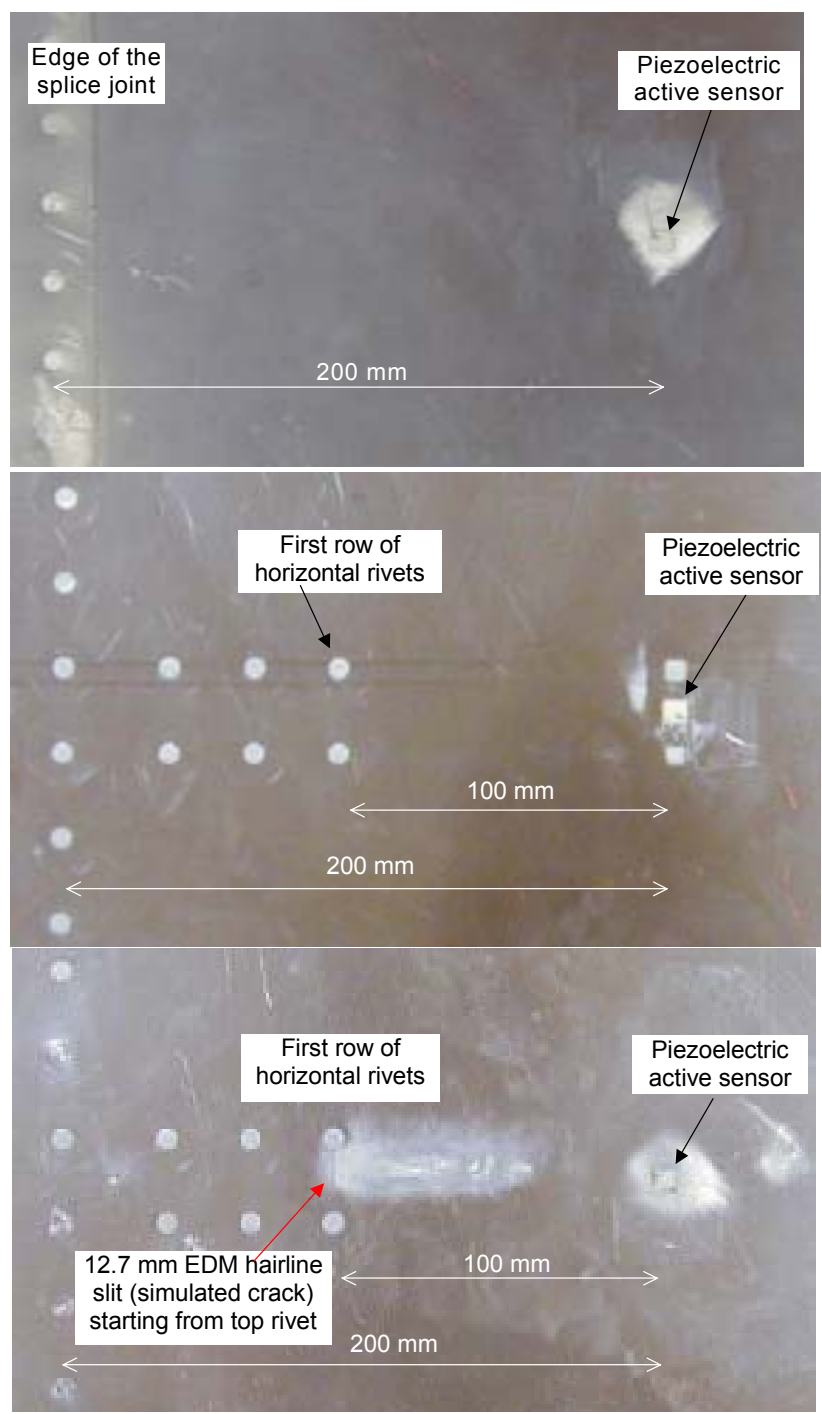

Figure 6 Crack-detection laboratory experiments on an aircraft panel: left column represents specimens (40-mil 2025 T3) with increasing complexity. Right column represents the pulseecho signals. Fourth cell in the right column shows the crack detection through the differential signal method.

In practice, such a situation would correspond to subtracting a signal previously recorded on the undamaged structure from the signal recorded now on the damaged structure. Such a situation of using archived signals is typical of health monitoring systems. When the two signals were subtracted, the result presented in the last row of Figure 6 was obtained. This differential signal shows a "loud and clear" echo due entirely to the crack. The echo,
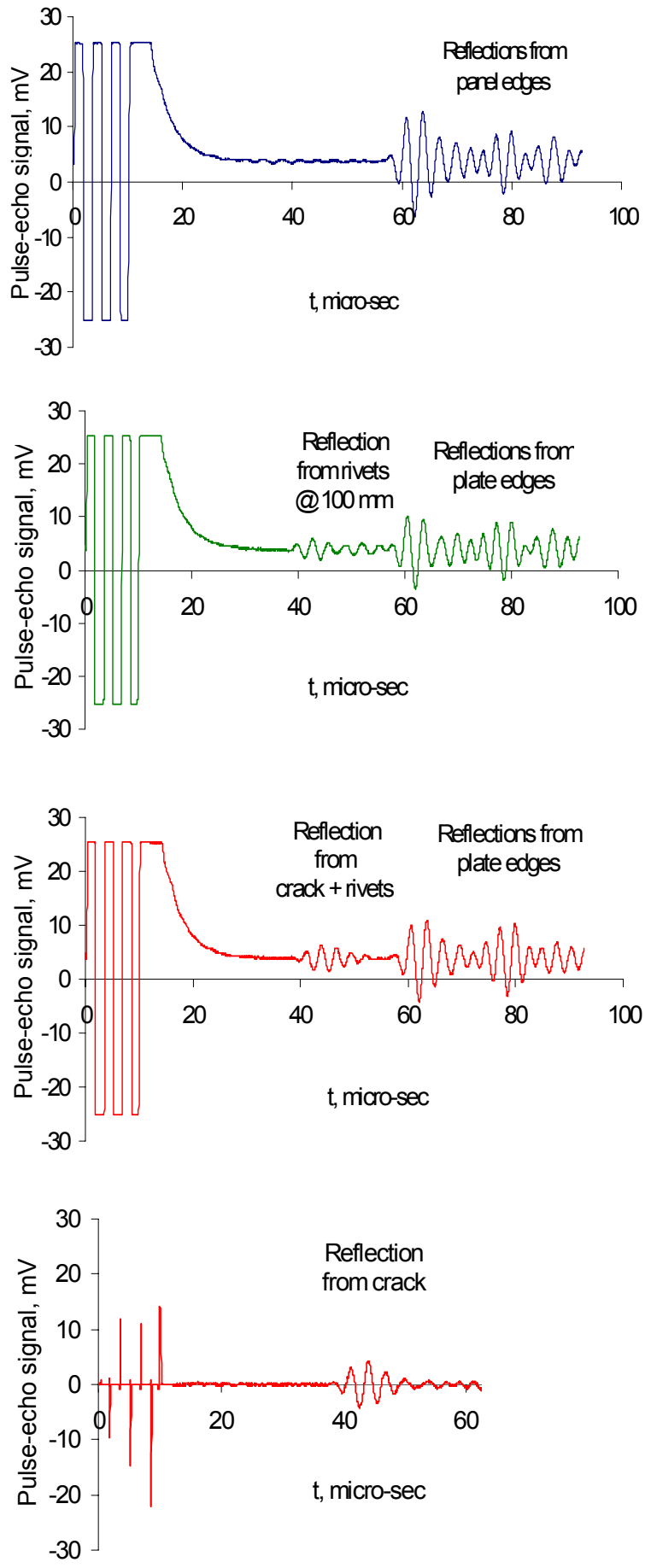

marked "reflection from the crack" is centered at 42 $\mu$ s, i.e., $\mathrm{TOF}=37 \mu$ s wich correlates very well with a $5.4 \mathrm{~km} / \mathrm{s} 200-\mathrm{mm}$ total travel from the PWAS to the crack placed at $100 \mathrm{~mm}$. The cleanness of the crackdetection feature and the quietness of the signal ahead of the crack-detection feature are remarkable. Thus, we concluded that PWAS are capable of clean and un-ambiguous detection of structural cracks. 


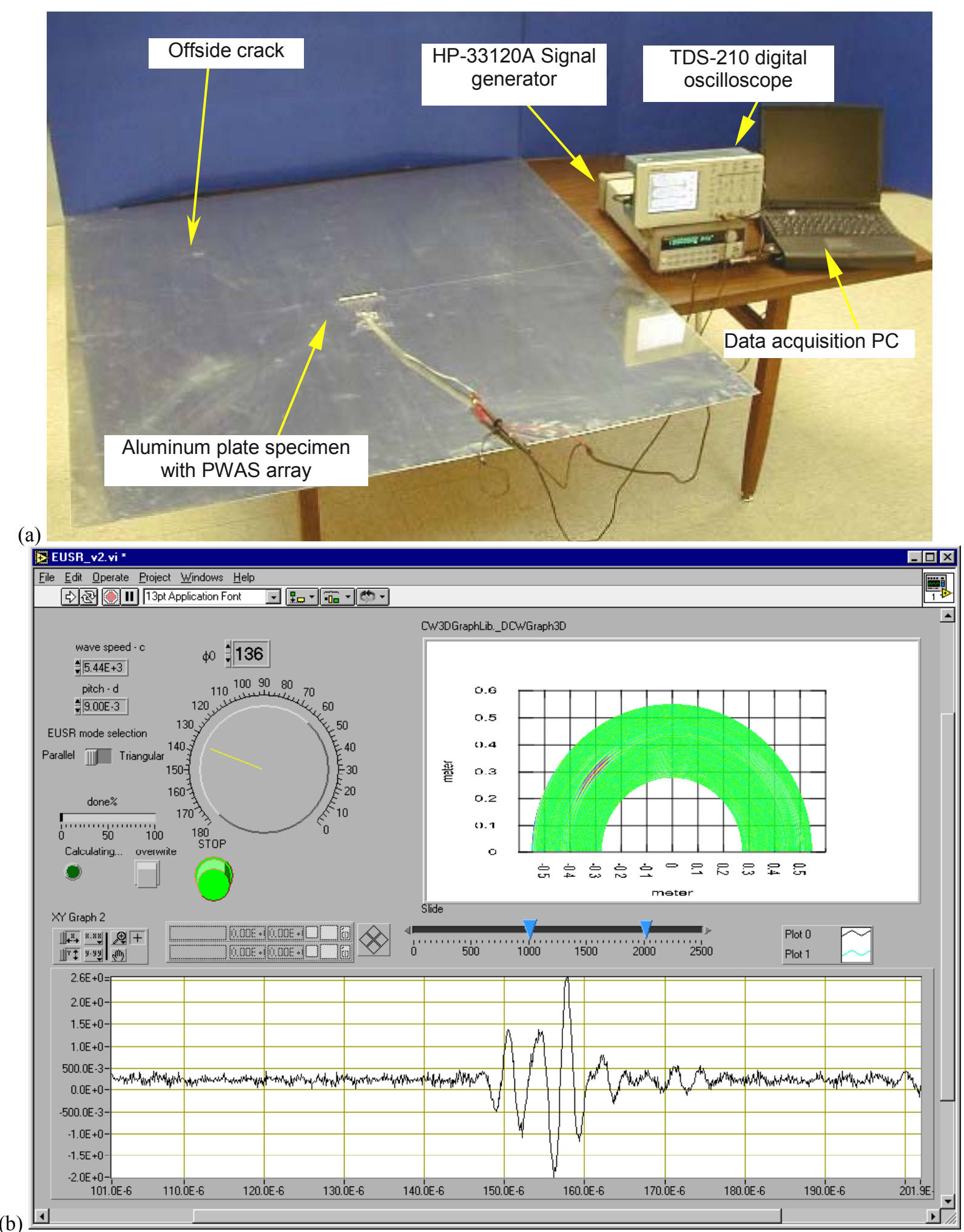

Figure 7 - Proof-of-concept EUSR experiment: (a) thin plate specimen 9-element PWAS array and 19-mm offside crack; (b) Graphical user interface (EUSR-GUI) front panel. The angle sweep is performed automatically to produce the structure/defect imaging picture on the right. Manual sweep of the beam angle can be also performed with the turn knob; the signal reconstructed at the particular beam angle (here, $\phi_{0}=136$ deg) is shown in the lower picture. 


\section{PWAS PHASED ARRAYS}

The advantages of phased array transducers for ultrasonic testing are multiple ${ }^{18,19}$. Krautkramer, Inc. produces a line of phased array transducers for the inspection of very thick specimens, and in the sidewise inspection of thick slabs, etc. These transducers employ pressure waves generated through normal impingement on the material surface.

In our research ${ }^{20}$, we have developed a phased array technology for thin wall structures (e.g., aircraft shells, storage tanks, large pipes, etc.) that uses Lamb waves to cover a large surface area through beam steering from a central location. We called this concept embedded ultrasonics structural radar (EUSR) and constructed a simple proof-of-concept experiment (Figure 7a). A PWAS array was made up of a number of identical 7-mm sq. elements aligned at uniform 9$\mathrm{mm}$ pitch. The PWAS phased array was placed at the center of a $4-\mathrm{ft}$ square thin aluminum plate (Figure $7 \mathrm{a}$. The wave pattern generated by the phased array is the result of the superposition of the waves generated by each individual element. By sequentially firing the individual elements of an array transducer at slightly different times, the ultrasonic wave front can be focused or steered in a specific direction. Thus, we achieved electronic sweeping and/or refocusing of the beam without physical manipulating the transducers. We proved that inspection of a wide zone is possible by creating a sweeping beam of ultrasonic Lamb waves that covered the whole plate. Once the beam steering and focusing was established, the detection of crack was done with the pulse-echo method.

During these proof-of-concept experiments, the EUSR methodology was used to detect cracks in two typical situations: (i) a 19-mm broadside crack placed at 305 $\mathrm{mm}$ from the array in the $90 \mathrm{deg}$ direction; and (ii) a $19-\mathrm{mm}$ broadside crack placed at $409 \mathrm{~mm}$ from the array in the $136 \mathrm{deg}$ direction. Of these two, the latter was more challenging because the ultrasonic beam is not reflected back to the source but rather deflected sideways. Hence, the echo received from the offside crack is merely the backscatter signal generated at the crack tips. Figure $7 \mathrm{~b}$ presents the front panel GUI of the embedded ultrasonic structural radar graphical user interface (EUSR-GUI) displaying the offside signals. The sweep is performed automatically to produce the structural defect image in the right pane. Manual sweep can be performed with the turn knob. The reconstructed signal is shown in the lower pane. In Figure 7b, the lower pane show the signal reconstructed at the beam angle $\phi_{0}=136 \mathrm{deg}$ corresponding to the crack location.

\section{ELECTROMECHANICAL IMPEDANCE METHOD}

PWAS-generated Lamb standing waves are used in conjunction with the electro-mechanical (E/M) impedance technique for local-area structural damage detection. The electro-mechanical (E/M) impedance technique permits the direct measurement of the high$\mathrm{kHz}$ drive-point mechanical impedance spectrum as seen by a piezoelectric wafer active sensor (PWAS) mounted on the examined structure. The excitation at hundreds of $\mathrm{kHz}$ generates standing Lamb waves that represent localized structural modes that are highly sensitive to incipient damage. In addition, due to the high $\mathrm{kHz}$ bandwidth, such spectra are impervious to noise and disturbances originating from normal aircraft operation, because such noise and disturbances characteristically happen at most in the low $\mathrm{kHz}$ range. For this reason, the $\mathrm{E} / \mathrm{M}$ impedance method offers good opportunities for identification of incipient local damage during the vehicle structural health monitoring process.

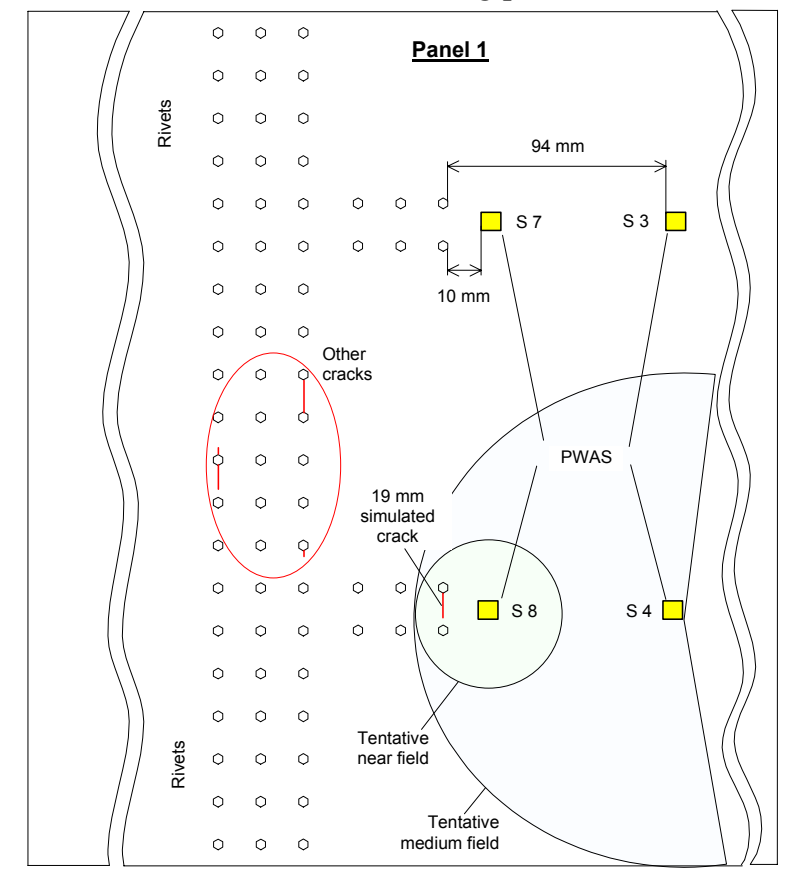

Figure 8 Panel 1 specimen and location of PWAS \# S3, S4, S7, S8

Figure 8 illustrates damage detection on a realistic aircraft panel with simulated cracks. In these experiments, we used two aircraft panels, one pristine (Panel 0), and the other with simulated cracks originating from rivet holes (Panel 1). The panels present typical structural elements (rivets, splices, stiffeners, etc.). Their presence complicates the structural dynamics and makes the damage detection task more difficult. The objective of the experiment was to detect a $19 \mathrm{~mm}$ simulated crack originating from a 
rivet hole. The panels were instrumented with eight piezoelectric wafer active sensors (PWAS), four on each panel: two sensors were placed in the medium field (100 $\mathrm{mm}$ from the crack location), and two in the near field $(10 \mathrm{~mm}$ from the crack location). The location of the sensors was identical on both panels. The sensor labels are given in Table 1. The damage detection was done from the $\mathrm{E} / \mathrm{M}$ impedance spectrum using probabilistic neural networks.

Table 1 Position on sensors on aircraft panels

\begin{tabular}{|c|c|c|c|c|}
\hline \multirow{2}{*}{} & \multicolumn{2}{|c|}{ Panel 0 } & \multicolumn{2}{c|}{ Panel 1 } \\
\cline { 2 - 5 } & Pristine & Pristine & Pristine & Crack \\
\hline Medium field & S1 & S2 & S3 & S4 \\
\hline Near field & S5 & S6 & S7 & S8 \\
\hline
\end{tabular}

The results of the PNN classification are presented in Table 2. Three tests were performed. In each test, one 'pristine' vector was used for training, and then the remaining three vectors were used for validation. Dichotomous classification was used: an outcome of 1 signifies that the vector presented during the validation was in the same class with the vector used during training, while an outcome of 0 signifies the opposite. Thus, all the vectors that return an outcome of 1 could be considered to represent 'pristine' conditions, while those that returned an outcome of 0 represented 'damage' conditions.

Table 2 Damage identification via probabilistic neural networsk classification of PWAS medium field and near field E/M impedance spectra using 48-frequencies features vectors

\begin{tabular}{|c|c|c|c|c|c|c|c|c|c|c|}
\hline \multirow{2}{*}{\multicolumn{2}{|c|}{ Vector }} & \multicolumn{4}{|c|}{ Medium Field } & \multicolumn{4}{|c|}{ Near Field } & \\
\hline & & S1 & $\mathrm{S} 2$ & S3 & $\mathrm{S} 4$ & S5 & S6 & $\mathrm{S} 7$ & S8 & \\
\hline \multirow{6}{*}{$\stackrel{\vec{w}}{\stackrel{0}{\varphi}}$} & \multirow{2}{*}{ I } & $\mathrm{T}$ & $\mathrm{V}$ & $\mathrm{V}$ & $\mathrm{V}$ & $\mathrm{T}$ & $\mathrm{V}$ & $\mathrm{V}$ & $\mathrm{V}$ & IN \\
\hline & & - & 0 & 0 & 1 & - & 0 & 0 & 1 & OUT \\
\hline & \multirow{2}{*}{ II } & $\mathrm{V}$ & $\mathrm{T}$ & $\mathrm{V}$ & $\mathrm{V}$ & $\mathrm{V}$ & $\mathrm{T}$ & $\mathrm{V}$ & $\mathrm{V}$ & $\mathrm{IN}$ \\
\hline & & 0 & - & 0 & 1 & 0 & - & 0 & 1 & OUT \\
\hline & \multirow{2}{*}{ III } & $\mathrm{V}$ & $\mathrm{V}$ & $\mathrm{T}$ & $\mathrm{V}$ & $\mathrm{V}$ & $\mathrm{V}$ & $\mathrm{T}$ & $\mathrm{V}$ & IN \\
\hline & & 0 & 0 & - & 1 & 0 & 0 & - & 1 & OUT \\
\hline
\end{tabular}

For example, in Test I, vector S1 was used for testing, while S2, S3, S4 were used for validation. The outcomes on S2 and S3 were 1, i.e., S2 and S3 belong in the same class with $\mathrm{S} 1$, i.e., 'pristine'. The outcome for $\mathrm{S} 4$ was 0 , i.e., $\mathrm{S} 4$ does not belong in the same class with S1, i.e., it does not represent a 'pristine' condition, hence it represents a 'damaged' condition. Table 2 indicates that all the three tests performed gave perfect results, i.e., the 'pristine' situations (S1, S2, S3) and the 'damage' situation were correctly classified irrespective of the training vector choice. After the successful classification of the medium-field data, the same PNN approach was also successfully used to perform the classification of the near-field data (second part of Table 2). Further details of this work can be found in ref. 21 .

\section{PWAS AS PASSIVE SENSORS}

PWAS can be also used as passive sensors, to detect the elastic Lamb waves transmitted into the structure by a low-velocity impact or an acoustic emission event.

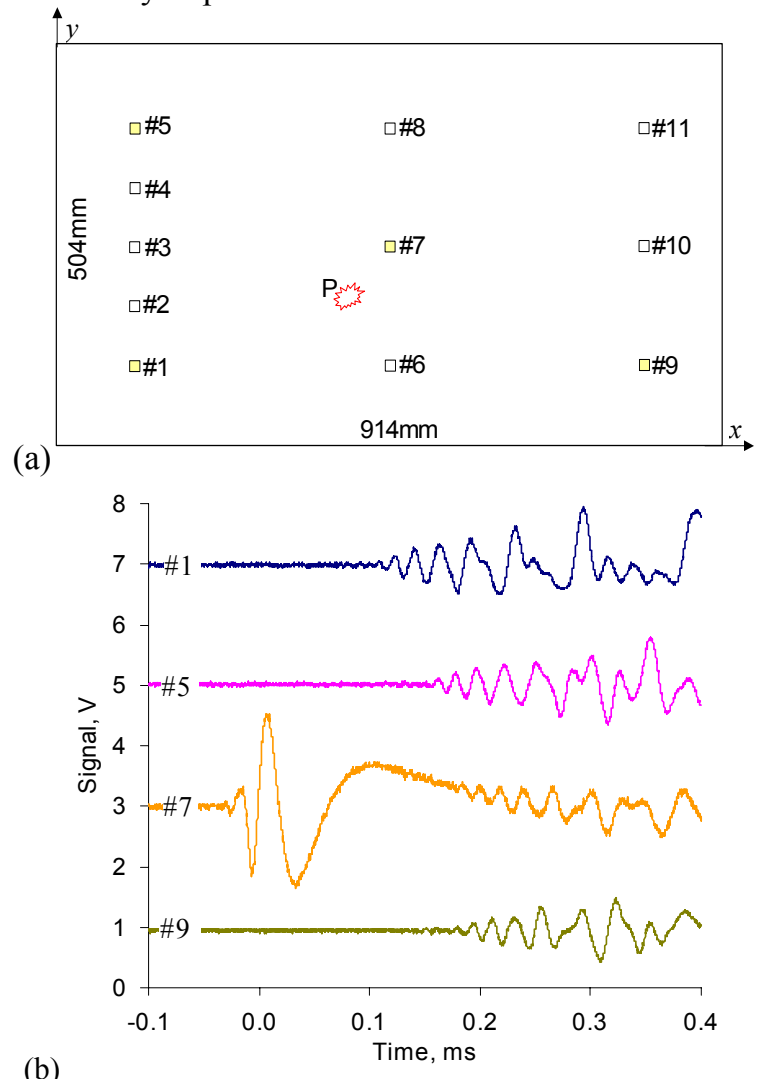

Figure 9 Impact detection (ID) experiments: (a) positioning of the impact relative to the sensors (b) captured ID signals

Impact Detection with Piezoelectric Wafer Active $\underline{\text { Sensors }}$

Impact detection with embedded piezoelectrics was successfully demonstrated in composite materials by Wang and Chang ${ }^{22}$. We used an array of PWAS on an aircraft-grade aluminum plate (Figure 9a). A small steel ball $(0.16 \mathrm{~g})$ was dropped from $50 \mathrm{~mm}$ height. The signals recorded on sensors $\# 1, \# 5, \# 7, \# 9$ are shown in Figure 9b (for display, the signals were spaced up by DC shifts). The high sensitivity of the PWAS devises is remarkable, since signals of up to $\pm 1.5 \mathrm{~V}$ were directly recorded without the need for any signal conditioning or pre-amplifiers. The corresponding TOF were $t_{1}=0.1070$ $\mathrm{ms}, t_{5}=0.1560 \mathrm{~ms}, t_{7}=-0.0312 \mathrm{~ms}, t_{9}=0.1800 \mathrm{~ms}$, relative to the oscilloscope trigger. A straight line fit $\left(\mathrm{R}^{2}\right.$ $=99.86 \%)$. through the distance-TOF points recovered the $\mathrm{A}_{0}$ group velocity of $c=1.606 \mathrm{~km} / \mathrm{s}$ at $45 \mathrm{kHz}$. 
Assuming the unknown impact position is $(x, y)$, the following set of simultaneous nonlinear equations represent the correlation between distance, group velocity and TOF:

$$
\left(x_{i}-x\right)^{2}+\left(y_{i}-y\right)^{2}=\left(c \cdot t_{i}\right)^{2}, \quad i=1, \ldots, 4
$$

This over-constrained set of equations was solved through error minimization and the impact location was determined as $x_{\text {impact }}=400.7 \mathrm{~mm}, y_{\text {impact }}=187.5$ $\mathrm{mm}$. These values are within $0.2 \%$ and $6.2 \%$, respectively, of the actual impact location $(400 \mathrm{~mm}$ and $200 \mathrm{~mm}$, respectively).

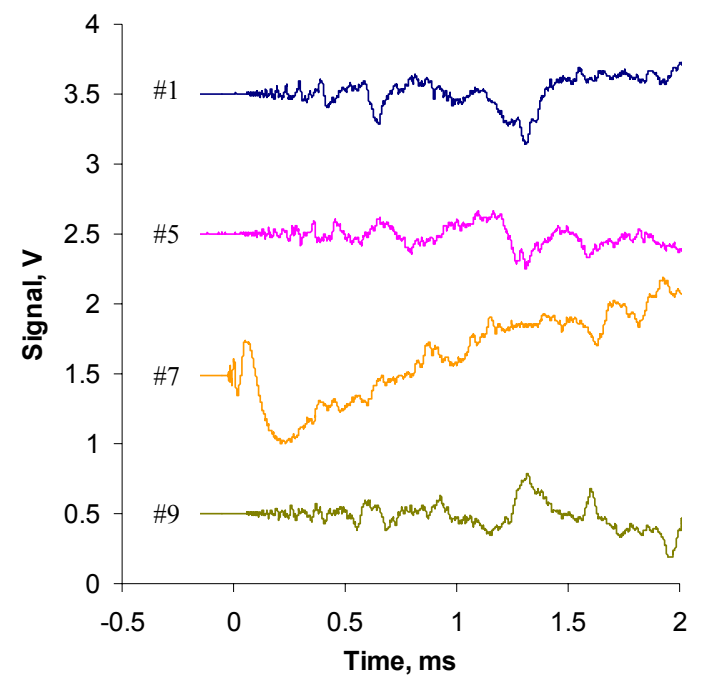

Figure 10 Signals captured from a simulated acoustic emission at $X_{P}=400 \mathrm{~mm}, y_{P}=200 \mathrm{~mm}$

\section{Acoustic Emission Detection with Piezoelectric Wafer Active Sensors}

Simulated acoustic-emission detection experiments were performed on the rectangular plate specimen of Figure 9a. Acoustic emission events were simulated at the location $\mathrm{P}, x_{\mathrm{P}}=400 \mathrm{~mm}, y_{\mathrm{P}}=200 \mathrm{~mm}$ using a pencil lead breaks on the specimen surface $(0.5 \mathrm{~mm}$ HB leads). This simulation method is consistent with that used by other investigators ${ }^{23,24,25}$.

The simulated acoustic emission signals captured at sensors $\# 1, \# 5, \# 7, \# 9$ are shown in Figure 10. The high sensitivity of these sensors is remarkable: signals of up to $\pm 0.5 \mathrm{~V}$ were directly recorded without the need for any signal conditioning or pre-amplifiers. The signal on sensor \#7, closest to the AE source $\left(r_{7}=\right.$ $71 \mathrm{~mm}$ ), is the strongest. This signal displays both high-frequency and low-frequency components, corresponding to $\mathrm{S}_{0}$ (axial) and $\mathrm{A}_{0}$ (flexural) waves, respectively. The flexural $\left(\mathrm{A}_{0}\right)$ waves display much higher amplitudes than the axial $\left(\mathrm{S}_{0}\right)$ waves, though they are dispersive and their travel speed is much slower.

\section{PWAS OPPORTUNITIES FOR VEHICLE STRUCTURAL \\ HEALTH MONITORING}

PWAS are a key component being examined to address the Air Force vision to localize incipient flaws, principally corrosion and cracking, in the complex geometric structure of aircraft. The Aloha Airlines Flight 811 that suffered the loss of a large section of its cabin fuselage while in flight (Figure 11) cemented a significant government focus on early crack detection in structural joints. Most effort to date has been directed at increasing the sensitivity of existing inspection tools and developing rapid scanning platforms that allow fast automated scanning of large areas on the aircraft. Since significant improvements have already been made in this area, the next frontier to address in this area is the use of on-board health monitoring.

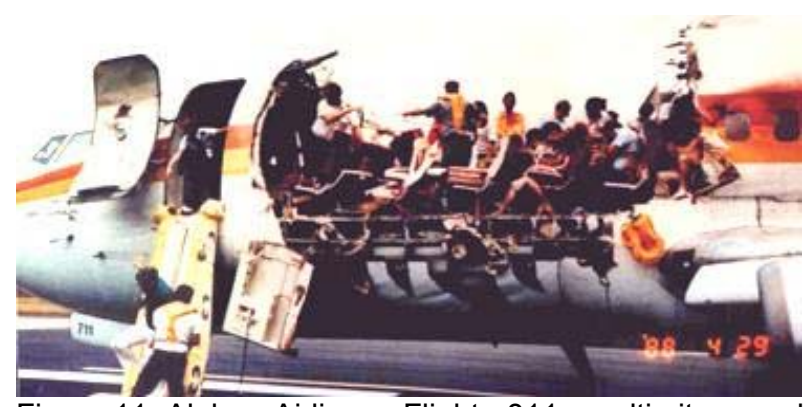

Figure 11 Aloha Airlines Flight 811 multi-site crack damage torn away the top of the aircraft skin

Structural health monitoring that provides owners and maintainers of long life expectancy vehicles is being envisioned to localize potential problem areas in the acreage of aircraft skin and underlying structure (Figure 12). This knowledge of evolving "hot spots" will then be used to target inspection techniques that will allow detailed characterization of the structure, which can be used to determine the appropriate maintenance and/or repair needs.

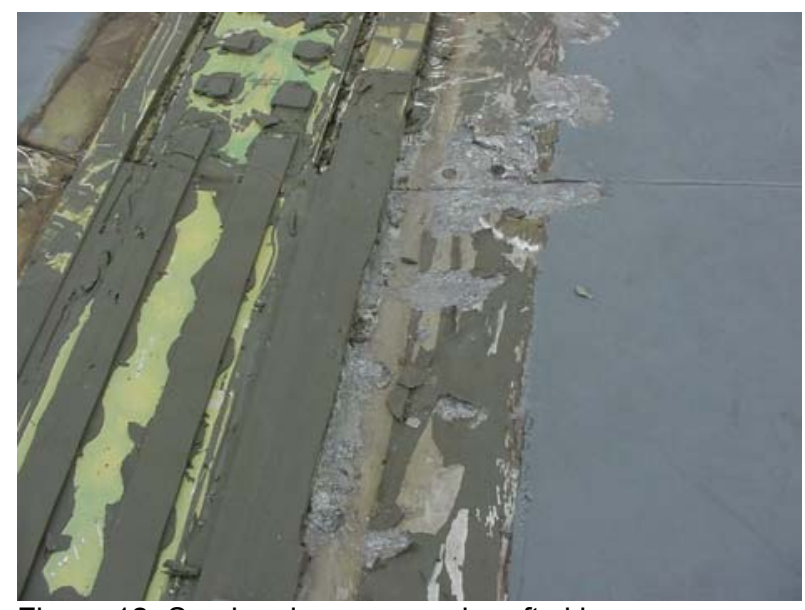

Figure 12 Service damage on aircraft skin 
This health monitoring approach has the advantage of a long-term change in maintenance philosophy. The current scheduled maintenance approach that relies on a "find and fix" process will evolve into a demand driven "fix when required" approach. The current Air Force depot maintenance costs exceed $\$ 13 \mathrm{~B}$ per year; several studies into demand driven maintenance concepts have predicted savings of at least $10 \%$ and potentially much more.

One conceptual vision for a structural health monitoring system is that of an artificial central nervous system. PWAS are seen as leading candidates to serve as one specialized type of "neuron" in this systems level concept.

\section{RESEARCH NEEDS IN PWAS-BASED STRUCTURAL}

\section{HEALTH MONITORING AND EMBEDDED NDE}

Many aspects of the use and usability of PWAS still need to be investigated. In a long-term perspective, the entire range of aircraft structure will eventually be instrumented with sensors and a key goal for PWAS is to determine where and how they will perform most effectively. Early investigations are focused on aircraft skin and lap joints. A majority of aircraft skin in the existing fleet ranges from 0.040 " to 0.065 " thick in alloys of 7075-T6 and 2024 aluminum, though many other alloys less common and some very thick skin exists in certain locations. Lap joint configurations vary widely from standard two layer configuration (with or without sealant) towards much more complex joints with multiple layers that may include one or more doubler (Figure 13). Rivets, screws and fasteners of may types are used to bind these lap joints and many joints contain attachment to various structural components such as ribs, spars, bulkheads, etc. The growth in the use of organic composites in aircraft structure introduces a whole new set of variables for health monitoring. Determining how PWAS can be used in these structures to detect fatigue cracking, corrosion growth (particularly in the advent of $>10 \%$ material loss), and stress corrosion cracking is the first step in this large area of research. It seems intuitive that PWAS will not be the optimal sensing tool for every case of structural monitoring: an understanding of which facet of this problem is suited for PWAS is required.

Another key aspect of structural health monitoring involves sensors that will have a very long useful life in service. It is anticipated that PWAS will be installed into an aircraft during depot maintenance activities when much of the skin is removed for inspection and repair. The typical time between depot maintenance can run from 6 to 8 years, so sensor durability and survivability are critical. Air turbulence and buffet will cause significant vibration and repetitive stress to the PWAS. Temperature swings from lows of $-40^{\circ} \mathrm{F}$ to highs of $150^{\circ} \mathrm{F}$ are possible. Corrosive effects of salts, de-icing agents, and many types fuels, fluids and lubricants are also present. Clearly, surviving in an operational environment is no small challenge.

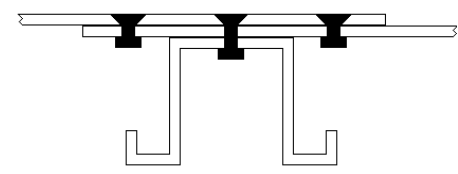

Figure 13 Typical aircraft lap-joint splice

Lastly, the usability of these sensors and their associated support systems, including power, network connectivity, and control systems must be integrated into the operational aircraft. Once all these hurdles have been addressed, PWAS will be ready for implementation into the Air Force's Fleet.

\section{CONCLUSIONS}

A novel structural health-monitoring concept embedded NDE with piezoelectric wafer active sensors (PWAS) has presented. PWAS can be structurally embedded as both individual probes and phased arrays. They can be placed even inside closed cavities during fabrication/overhaul (such as wing structures), and then be left in place for the life of the structure. The embedded NDE concept opens new horizons for performing in-situ damage detection and structural health monitoring of a multitude of thin-wall structures.

Conclusive experiments have been presented that verified that "loud and clear" ultrasonic Lamb waves can be successfully generated with these small, inexpensive, and unobtrusive devices. Wave omnidirectionality and long distance propagation were proven. The pulse-echo crack-detection method was demonstrated on a simple plate specimen and on a realistic aircraft panel. The arrangement of PWAS in phased arrays has opened additional opportunities, by permitting the sweeping of large areas from a central location. Successful detection of broadside and offside cracks in a large plate was illustrated with a simple-to-use graphical user interface (EUSR-GUI).

It ha bees shown that, with the same PWAS installation, four structural health-monitoring functions will be achieved:

(a) Active damage detection based on wave propagation (pulse-echo and pitch-catch)

(b) Active damage detection based on local impedance measured at hundreds of $\mathrm{kHz}$

(c) Low-velocity impact detection

(d) Acoustic emission detection

This multiple-use capability of PWAS networks opens important opportunities for their use in smart vehicle structural health monitoring, damage detection, and failure prevention. The paper has examined how these 
opportunities address the Air Force vehicle structural health monitoring needs, and what areas of research need to be addressed in more depth. This emerging technology requires a sustained R\&D effort to achieve its full developmental potential for applicability to full-scale aerospace vehicles.

\section{ACKNOWLEDGMENT}

2002 Summer Faculty Fellowship Program at the Air Force Research Laboratory NDE Branch, WrightPatterson AFB, OH.

\section{REFERENCES}

1. Kropas-Hughes, C.V.; Perez, I.; Winfree; W. P.; Motzer, W. P.; Thompson, R. B. (2002) "Vision of Future Directions of NDE Research" in Review of Quantitative Nondestructive Evaluation Vol. 21, ed. by D. O. Thompson and D. E. Chimenti, American Institute of Physics, Vol. 615, 2002, pp. 2042-2051

2. Rose, J. L. (2002) "A Baseline and Vision of Ultrasonic Guided Wave Inspection Potential", ASME Journal of Pressure Vessel Technology - Special Issue on Nondestructive Characterization of Structural Materials, Vol. 124, No. 3, August 2002, pp. 273-282

3. Viktorov, I. A. (1967) Rayleigh and Lamb Waves, Plenum Press, New York, 1967

4. Rose, J. L. (1999) Ultrasonic Waves in Solid Media, Cambridge University Press, 1999

5. Dalton, R. P.; Cawley, P.; Lowe, M. J. S. (2001) "The Potential of Guided Waves for Monitoring Large Areas of Metallic Aircraft Structure", Journal of Nondestructive Evaluation, Vol. 20, pp. 29-46, 2001

6. Thomson, D. O. and Chimenti, D. E. (Editors) (2002) Review of Progress in Quantitative Nondestructive Evaluation, Chapter 2C "Guided Waves" and Chapter 7 "NDE Applications", AIP Conference Proceedings Vol. 615, 2002

7. Keilers, C. H.; Chang, F.-K. (1995) "Identifying Delamination in Composite Beam using Built-in Piezoelectrics", Journal of Intelligent Material Systems and Structures, Vol. 6, 1995, pp. 647-672

8. Chang, F.-K. (2001) "Structural Health Monitoring: Aerospace Assessment", Aero Mat 2001, 12th ASM Annual Advanced Aerospace Materials and Processes Conference, 12-13 June 2001, Long Beach CA.

9. Lin, X.; Yuan, F. G. (2001) "Diagnostic Lamb Waves in an Integrated Piezoelectric Sensor/Actuator Plate: Analytical and Experimental Studies", Smart Materials and Structures, Vol. 10, 2001, pp. 907-913

10. Giurgiutiu, V.; Zagrai, A. (2000) "Characterization of Piezoelectric Wafer Active Sensors", Journal of Intelligent Material Systems and Structures, Technomic Pub., USA, Vol. 11, No. 12, December 2000, pp. 959-976

11. Giurgiutiu, V.; Zagrai, A. N.; Bao, J. (2002) "Embedded Active Sensors For In-Situ Structural Health Monitoring of Thin-Wall Structures", ASME Journal of Pressure Vessel Technology, Vol. 124, No. 3, August 2002, pp. 293-302

12. Diamanti, K.; Hodgkinson, J.M.; Soutis, C. (2002) "Damage Detection of Composite Laminates Using PZT Generated Lamb Waves", 1st European Workshop on Structural Health Monitoring, July 10-12, 2002, Paris, France, pp. 398-405

13. Ghoshal, A.; Martin W.N.; Schulz, M.J. (2002) "Simulation of
Asymmetric Lamb Wave Propagation for Health Monitoring", 1st European Workshop on Structural Health Monitoring, July 10-12, 2002, Paris, France, pp. 365-373

14. Guy, P.; Monnier, T.; Baboux, J.C.; Jayet Y. (2002) "Simultaneous Monitoring of Aging and Damage in Plate-Like Composite Structures Using Integrated Piezoelectric Sensors" 1st European Workshop on Structural Health Monitoring, July 10-12, 2002, Paris, France, pp. 352-358

15. Peairs, D.M.; Park, G.; Inman, D.J. (2002) "Low Cost Impedance Monitoring Using Smart Materials" 1st European Workshop on Structural Health Monitoring, July 10-12, 2002, Paris, France, pp. $442-449$

16. Giurgiutiu, V.; Zagrai, A. N. (2002) "Embedded Self-Sensing Piezoelectric Active Sensors for Online Structural Identification", ASME Journal of Vibration and Acoustics, Vol. 124, January 2002, pp. 116-125

17. Wallbrink, C.; Jones, R.; Tan, M.; Dayawansa, D. (2002) "Structural Health Monitoring of Dragline Structures Using Ultrasonic Waves" 1st European Workshop on Structural Health Monitoring, July 10-12, 2002, Paris, France, pp. 382-389

18. Krautkramer, J.; Krautkramer, H. (1990) Ultrasonic Testing of Materials, Springer-Verlag, 1990

19. Lines D.; Dickson K. (1999) "Optimization of High-Frequency Array Technology for Lap-Joint Inspection"; Proceedings of the 3rd Joint Conference on Aging Aircraft, 1999, www.galaxyscientific.com/agingaircraft/index2.htm

20. Giurgiutiu, V.; Bao, J. (2002) "Embedded Ultrasonic Structural Radar for Nondestructive Evaluation of Thin-Wall Structures" Proceedings of the 2002 ASME International Mechanical Engineering Congress, November 17-22, 2002, New Orleans, LA, paper \# IMECE2002-39017

21. Giurgiutiu, V. (2002) "Damage Metric Algorithms for ActiveSensor Structural Health Monitoring", 1st European Workshop on Structural Health Monitoring, July 10-12, 2002, Paris, France, pp. 433-441

22. Wang, C. S.; Chang, F.-K. (2000) "Built-In Diagnostics for Impact Damage Identification of Composite Structures", in Structural Health Monitoring 2000, Fu-Kuo Chang (Ed.), Technomic, 2000, pp. $612-621$

23. Blanas, P., Wenger, M. P., Shuford, R. J., Das-Gupta, D. K. (1997) "Active Composite Materials and Damage Monitoring", International Workshop on Structural Health Monitoring, Stanford University, CA, September 18-20, 1997, pp. 199-207.

24. Prosser, W. H.; Gorman, M. R. (1994) "Accurate Simulation of Acoustic Emission Source in Composite Plates", ASNT 1994 Third Annual Research Symposium, New Orleans, LA, March 21-25, 1994, pp. 152-154

25. Dzenis, Y.; Qian, J. (2001) "Analysis of Microdamage Evolution Histories in Composites", $7^{\text {th }}$ ASME NDE Topical Conference, San Antonio, TX, April 23-25, 2001. 